\title{
ALTERED LIPID PROFILE IN HYPOTHYROIDISM: A BIOCHEMICAL VIEWPOINT
}

\author{
KRISHNA VENI DV ${ }^{1}$, NEETHA KUNDOOR ${ }^{1}$, SRILATHA BASHETTI ${ }^{1}$, PRASHNATHI RAYAPROLU ${ }^{2}$
}

${ }^{1}$ Department of Biochemistry, Apollo Institute of Medical Sciences and Research, Hyderabad, Telangana, India. ${ }^{2}$ Department of Biochemistry, Bhaskar Medical College, Hyderabad, Telangana, India. Email: Dr.dvkvenibio@gmail.com

Received: 14 December 2017, Revised and Accepted: 11 April 2018

ABSTRACT

Context: Overt hypothyroidism is the most common endocrine disorder associated with a decrease in 3,5,3'-triiodothyronine and 3,5,3',5'-tetraiodothyronine levels and increase in thyroid-stimulating hormone (TSH) levels. Thyroid hormones (THs) regulate all major metabolic pathways. THs play an important role in the synthesis, metabolism, and transport of lipids. Thyroid failure is associated with various biochemical and molecular alterations. Hypothyroidism is the common cause of secondary dyslipidemia as well as cardiovascular risk.

Objective: The present review focuses on alterations in lipid metabolism associated with hypothyroidism leading to cardiovascular risk.

Methods: The data source is from PUBMED, Google Scholar, and EMBASE. The study is a part of our previous research in studying the variations in lipid profile and transaminases in overt hypothyroidism.

Result: The serum total cholesterol, low-density lipoprotein cholesterol (LDL-C), apolipoprotein B (apo B), lipoprotein (a) (Lp[a]), and triglycerides are elevated in individuals with overt hypothyroidism. In addition, it was also observed that the endothelial dysfunction, normal/depressed systolic function, left ventricular diastolic dysfunction at rest, as well as systolic and diastolic dysfunction on effort was observed in hypothyroidism.

Conclusion: THs play an important role in the regulation of lipid metabolism. Hypothyroidism is also linked with altered hemodynamics. The atherogenic lipid profile along with other predisposing factors may contribute to the coronary artery disease.

Keywords: Hypothyroidism, Lipid profile, Cardiovascular disease, Lipoproteins.

(C) 2018 The Authors. Published by Innovare Academic Sciences Pvt Ltd. This is an open access article under the CC BY license (http://creativecommons. org/licenses/by/4. 0/) DOI: http://dx.doi.org/10.22159/ajpcr.2018.v11i7.24277

\section{INTRODUCTION}

Hypothyroidism is a common endocrine disorder, which is diagnosed by estimating the thyroid hormone (TH) levels in the blood and effectively treated by the daily use of synthetic THs [1]. The prevalence of hypothyroidism according to the third National Health and Nutrition Examination Survey III is $4.6 \%$ in the general population, whereas 9.5 $\%$ of Colorado prevalence study participants showed elevated levels of thyroid-stimulating hormone (TSH) [2]. Hypothyroidism is prevalent in older women, in whom autoimmune thyroiditis is common [1]. Hypothyroidism may be due to inadequate synthesis of $\mathrm{TH}$ or inadequate action of TH at the target tissue level [3]. Hypothyroidism ranges from mild subclinical form to overt hypothyroidism and myxedema. Depending on the time of onset hypothyroidism can be classified as congenital or acquired, depending on the level of endocrine dysfunction as primary and secondary or central hypothyroidism [1]. As thyroid function diminishes, serum TSH level begins to rise. An elevated serum TSH level is the hallmark of hypothyroidism. Subclinical hypothyroidism ( $\mathrm{SCH}$ ) or mild thyroid failure is associated with elevated TSH but a normal free thyroxin tetraiodothyronine $\left(\mathrm{T}_{4}\right)$ and triiodothyronine $\left(\mathrm{T}_{3}\right)$ levels [4]. Severe iodine deficiency during pregnancy decreases fetal TH synthesis causes irrepairable damage to the fetal central nervous system, leading to mental retardation is termed as cretinism [5]. $\mathrm{T}_{3}$ is considered to be the metabolically active form [1]. Under normal physiological conditions, a major fraction of $\mathrm{T}_{3}$ is derived from the peripheral deiodination of $\mathrm{T}_{4}$ and only a lesser fraction is derived from direct secretion by the thyroid gland [6]

Patient presentation in hypothyroidism may vary from asymptomatic to myxedema coma. The classic symptoms and signs of hypothyroidism are weight gain, fatigue, cold intolerance, constipation, dry skin, hoarseness, goiter, mental impairment, depression, mild diastolic hypertension, narrowed pulse pressure, bradycardia, decreased appetite, or arthralgia [1]. Both overt and SCH, markedly alter lipid profile and promote cardiovascular disease (CVD) [6]. The aim of the present review is to illustrate the functions and metabolism of TH and to highlight alterations of lipid profile in hypothyroidism which may have a predisposing cardiovascular risk.

\section{Synthesis and secretion of THs}

The main function of the thyroid gland is to synthesize sufficient amount of THs to meet the demands of peripheral tissue. At least $100 \mu \mathrm{g}$ of iodine per day is required to eliminate the signs of iodine deficiency. Synthesis of THs includes iodine uptake, trapping, iodide oxidation, organification, and coupling of iodotyrosyls (Fig. 1). Iodide trapping is accomplished by a 643 amino acid membrane glycoprotein with 13 membrane-spanning domains, sodium-iodide symporter (NIS), and encoded by the gene SLC5A5 (Solute Carrier Family 5 Member 5). Iodide transport is an active process and depends on the presence of a sodium gradient across the basal membrane of the thyroid cell. The downhill transport of two $\mathrm{Na}^{+}$ ions results in the entry of one iodide atom against an electrochemical gradient. Another membrane glycoprotein Pendrin, which is highly hydrophobic, is located in the apical membrane of follicular cells and functions as an apical iodide transporter in thyroid cells. It is encoded by SLC26A4. Other proteins (SLC5A8 and chloride channel 5, CLCN5) have been proposed to mediate apical iodide efflux. In addition, intracellular iodide is also synthesized by iodotyrosine dehalogenase 1 , also called as iodotyrosine deiodinase. Its transcription is stimulated by cyclic adenosine monophosphate and encodes a membrane protein concentrated on the apical surface which catalyzes reduced nicotinamide adenine dinucleotide phosphate (NADPH)-dependent deiodination of monoiodotyrosine and diiodotyrosine (MIT and DIT).

Oxidation of iodide is mediated by the heme-containing protein thyroperoxidase (TPO) and requires $\mathrm{H}_{2} \mathrm{O}_{2}$ generated by the calcium 
and NADPH-dependent dual oxidase (DUOX) 1 and DUOX2 enzymes. DUOX maturation factor 2 is required for maturation and plasma membrane localization of DUOX2 and $\mathrm{H}_{2} \mathrm{O}_{2}$. The process of oxidation of iodide and incorporation into iodotyrosines (MIT and DIT) is termed as organification. This iodination leading to the synthesis of iodotyrosines occurs within thyroglobulins $\left(\mathrm{T}_{\mathrm{g}}\right)$. TPO also catalyzes coupling of two molecules of DIT or one of DIT and MIT leading to the formation of $\mathrm{T}_{4}$ and $\mathrm{T}_{3}$, respectively (Fig. 2). TPO is stimulated by TSH.
$\mathrm{T}_{4}$ and $\mathrm{T}_{3}$ are stored in the colloid as a part of $\mathrm{T}_{\mathrm{g}}$ [5]. From the follicular lumen colloid is taken in to the thyroid follicle by endocytosis ( microendocytosis ). The endocytic vesicles fuse with lysosomes. By the proteolytic action of lysosomal enzymes called cathepsin $\mathrm{D}$ and D like thiol proteases, MIT, DIT, T3 and T4 are released. MIT and DIT are metabolized by thyroid deiodinase to tyrosine and iodine which are recycled to the colloid and the iodide liberated is reincorporated into protein [7].

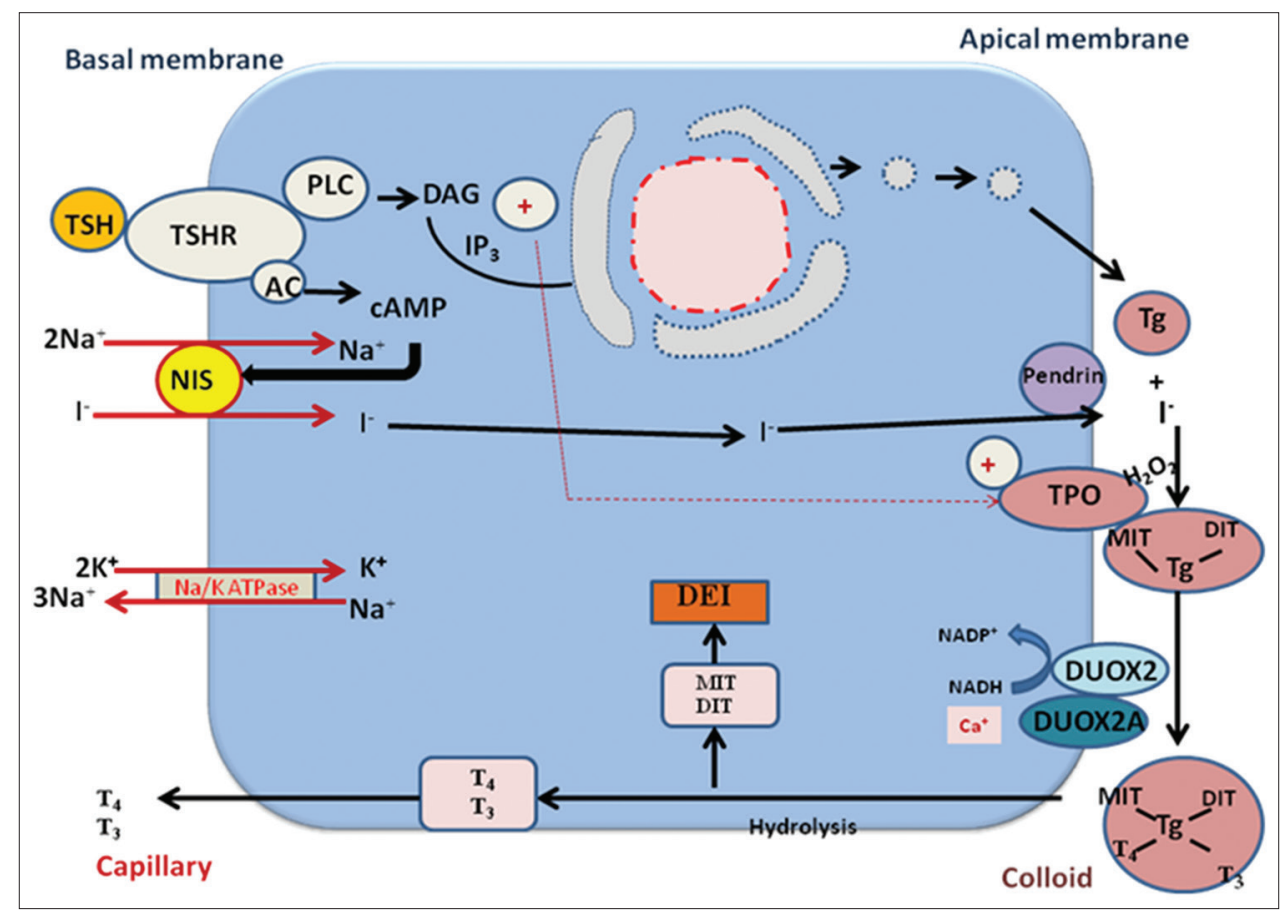

Fig. 1: Synthesis of thyroid hormones [7]. AC: Adenyl cyclase, TSH: Thyroid-stimulating hormone, TSHR: Thyroid-stimulating hormone receptor, PLC: Phospholipase C, DAG: Diacylglycerol, C AMP: Cyclic adenosine monophosphate, IP3: Inositol triphosphate, TPO: Thyroperoxidase, $\mathrm{H}_{2} \mathrm{O}_{2}$ : Hydrogen peroxide, DUOX2: Dual oxidase 2, DUOX2A: Dual oxidase maturation factor 2, MIT: Monoiodotyrosines, DIT: Diiodotyrosines, DEI: Deiodinase

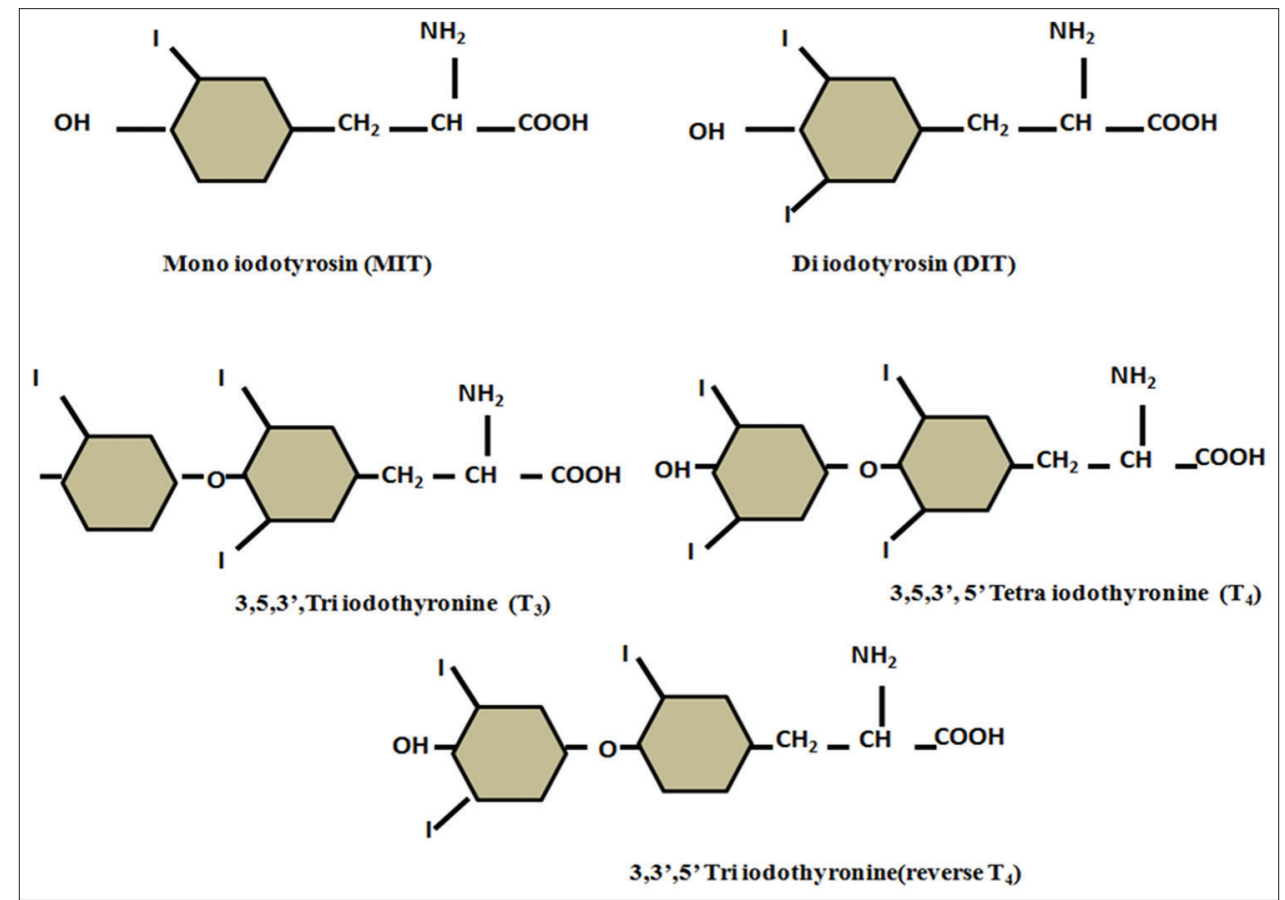

Fig. 2: Structure of monoiodotyrosine and diiodotyrosine, triiodothyronine, and tetraiodothyronine 
Hypothalamo-pituitary axis

The hypothalmo-pituitary-thyroid axis is a neuroendocrine system which regulates the secretion of THs. Thyrotropin-releasing hormone, which is secreted by the hypothalamus stimulates anterior pituitary to release TSH. TSH, in turn, stimulates the thyroid gland to secrete prohormone thyroxin $\left(\mathrm{T}_{4}\right)$ and to lesser extent $\mathrm{T}_{3}$ [8] (Fig. 3). $\mathrm{T}_{4}$ is converted into $\mathrm{T}_{3}$ which is metabolically active, by iodothyronine deiodinase. Most $\mathrm{TH}^{3}$ in the blood circulates bound to thyroid binding globulin, whereas only the free form has hormonal activity. There are specific cell membrane transporters such as monocarboxylate transporter 8 which help in transport of $\mathrm{TH}$ across the cell membrane. $\mathrm{T}_{3}$ interacts with nuclear receptors and activates or inactivates THs responsive gene [8].

\section{TH and lipid metabolism}

THs regulate the metabolism of lipoproteins (LP). Thyroid dysfunction is associated with various molecular and biochemical alterations. In hypothyroidism, the composition of LP and their transport is seriously disturbed. Hypothyroidism causes hypercholesterolemia characterized by increased levels of low-density lipoproteins (LDL) [9-11]. In addition to LP metabolism, thyroid function significantly affects CVD risk factors, thus influencing overall coronary artery disease (CAD) risk $[2,6]$. Hypothyroidism is a common cause of secondary dyslipidemia $[12,13]$. In a multicenter study, the prevalence of hypothyroidism was evaluated in 752 hypercholesterolemic patients; primary hypothyroidism amounted to $3.7 \%, \mathrm{SCH}$ to $2.4 \%$, and overt hypothyroidism to $1.4 \%$ [9]. The overall prevalence of hypothyroidism was calculated as $4.3 \%$ in patients with hypercholesterolemia [1]. The present review focuses on the impact of THs on lipid metabolism, which predisposes cardiovascular risk in hypothyroid patients.

\section{Overview of lipid metabolism}

Cholesterol synthesis is endergonic pathway, being driven by hydrolysis of the high-energy thioester bond of acetyl coenzyme A (COA) and

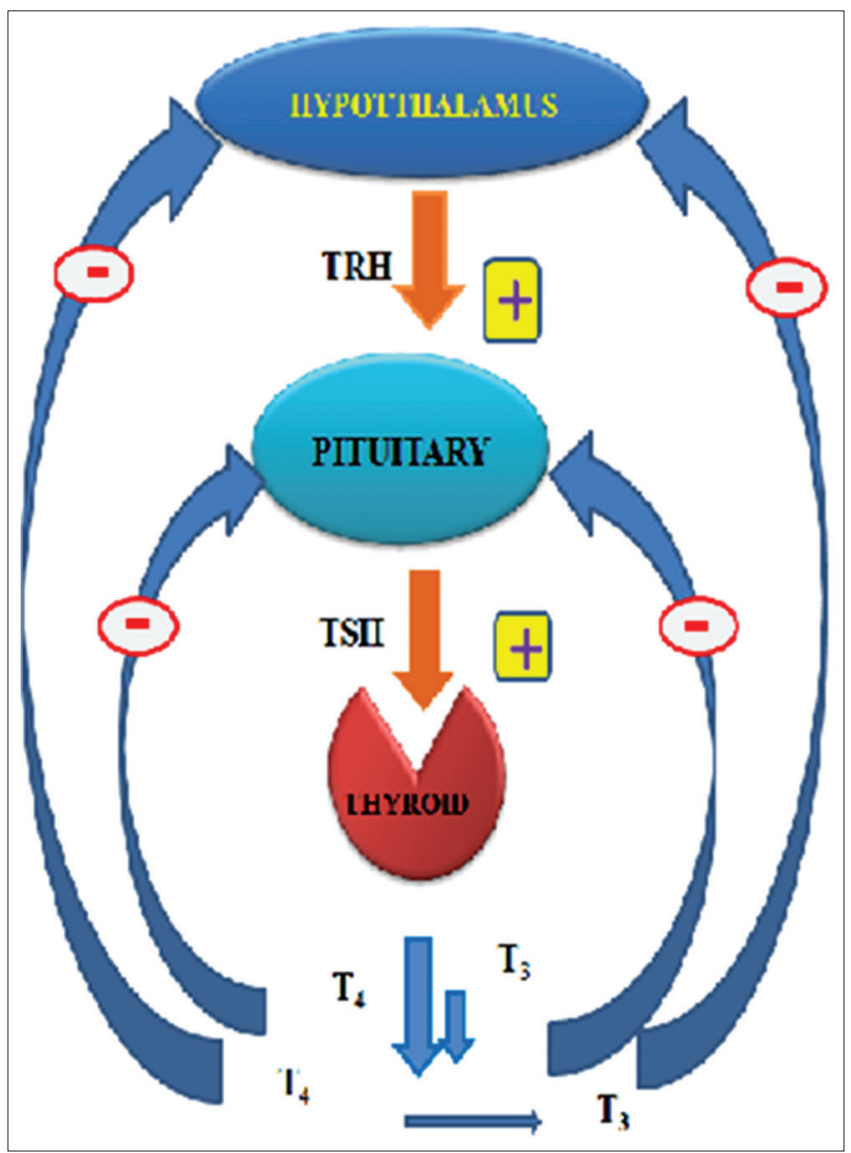

Fig. 3: Hypothalamus-pituitary axis [8] the terminal phosphate bond of adenosine triphosphate (ATP). The enzymes required for synthesis are present both in the cytoplasm and endoplasmic reticulum (ER). The first step in cholesterol is synthesis is condensation of two molecules of acetyl CoA forming acetoacetyl-CoA, which in turn by the addition of one more molecule of acetyl CoA, forms 3-hydroxy 3-methyl glutaryl-CoA (HMG-CoA). HMG-CoA is reduced to mevalonate by the rate-limiting enzyme HMG-CoA reductase, which undergoes a series of phosphorylations and cyclizations leading to the formation of squalene then finally cholesterol.

The main function of LPs is to keep their component lipids soluble as they transport them between tissues. Based on their density, the plasma LP molecules are classified into chylomicrons (CM), very LDL (VLDL), intermediate-density lipoproteins (IDL), LDL, and high-density lipoproteins (HDL). These LPs differ from each other in density, lipid and protein composition, size, and electrophoretic mobility. The CM transports exogenous or dietary fat and cholesterol, whereas VLDL transport endogenous triglycerides (TGs). Cholesterol was synthesized and secreted by the liver $[14,15]$.

TGs make up $90 \%$ of CMs and 75\% of VLDL by weight [14]. CMs and VLDL are hydrolyzed by LP lipase (LPL), an extracellular enzyme that is anchored by heparin sulfate to the capillary walls of most tissues, but predominantly those of adipose tissue and cardiac tissue. Thus, TG is hydrolyzed by LPL-producing smaller particles known as remnants [15]. The hepatic receptors, which contain apo (E), recognizes and removes the CMs remnants [16]. The dietary cholesterol from the chylomicron remnant particles is thought to downregulate the hepatic LDL receptors. VLDL remnants, also known as IDL, contain apo $\mathrm{E}$ and may be removed by the liver through the receptor-mediated endocytosis. The triacylglycerol concentration in LDL particles is less than their VLDL predecessor and cholesterol, and cholesterol ester concentration is more. LDL is the major cholesterol carrier, followed by HDL [15,17]. The chief function of LDL particles is to transport cholesterol to the peripheral tissue. The level of low-density lipoprotein cholesterol (LDL-C) regulated by the amount of LDL receptors. Defects in the LDL receptor molecule leads to hypercholesterolemia and myocardial infarction [16]. HDL particles take up cholesterol from the peripheral tissues and return it to the liver as cholesterol esters by the action of lecithin cholesterol acyltransferase (LCAT). HDL cholesterol is cardioprotective (Fig. 4).

\section{Reverse cholesterol transport}

Reverse cholesterol transport pathway includes efflux of cholesterol from peripheral cells to HDL, esterification of cholesterol by LCAT, binding of HDL-2 to the liver and steroidogenic cells, transfer of cholesterol esters into these cells, and release of HDL-3 $[17,18]$. The major constituents of reverse cholesterol transport are HDL, apolipoprotein A-1 (Apo-A1), enzymes like LCAT, phospholipid transfer protein, hepatic lipase (HL), and cholesterol ester transfer protein (CETP) [17-19] (Fig. 5). ATP-binding membrane cassette transporter (ABC-A1) mediates the efflux of cholesterol from the peripheral tissue and cell surface receptor and scavenger receptor class B Type 1 (SR-B1) mediates the uptake of cholesterol $[18,19]$.

Apolipoproteins, or apoproteins, are proteins associated with LP molecules. They have different functions such as providing a recognition site for cell-surface receptors or acts as activators or coenzymes for enzymes involved in LP metabolism. Apo B-100 is necessary for the secretion of hepatic-derived VLDL, IDL, and LDL. Apo B-48 is a truncated form of ApoB100, which is required for secretion of CMs from the small intestine. Apo-A1 is a major component of the HDL and acts as a cofactor for LCAT [14].

Cholesterol homeostasis is maintained by multiple feedback controls which act through transcriptional and post-transcriptional mechanisms. The transcription factors sterol regulatory elementbinding proteins (SREBP) play an important role in sterol synthesis and uptake. The SREBP are sterol-sensing transcription factors of the basic helix-loop-helix-leucine zipper (bHLH-Zip) family [20]. 


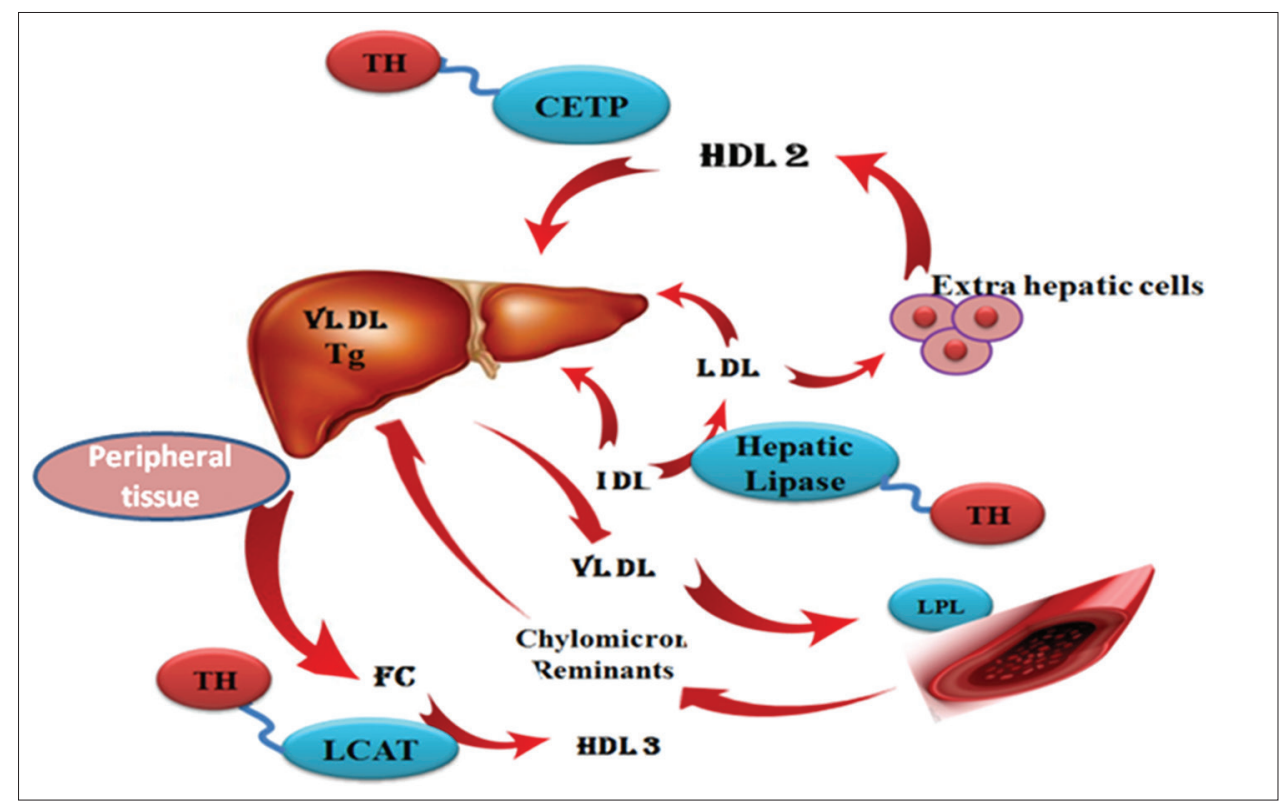

Fig. 4: Cholesterol transport [14]. TG: Triglycerides, HDL: High-density lipoproteins, LDL: Low-density lipoproteins, VLDL: Very lowdensity lipoproteins, IDL: Intermediate-density lipoproteins, TH: Thyroid hormones, CETP: Cholesterol ester transfer protein, LPL:

Lipoprotein lipase, FC: Free cholesterol, LCAT: Lecithin cholesterol transfer protein

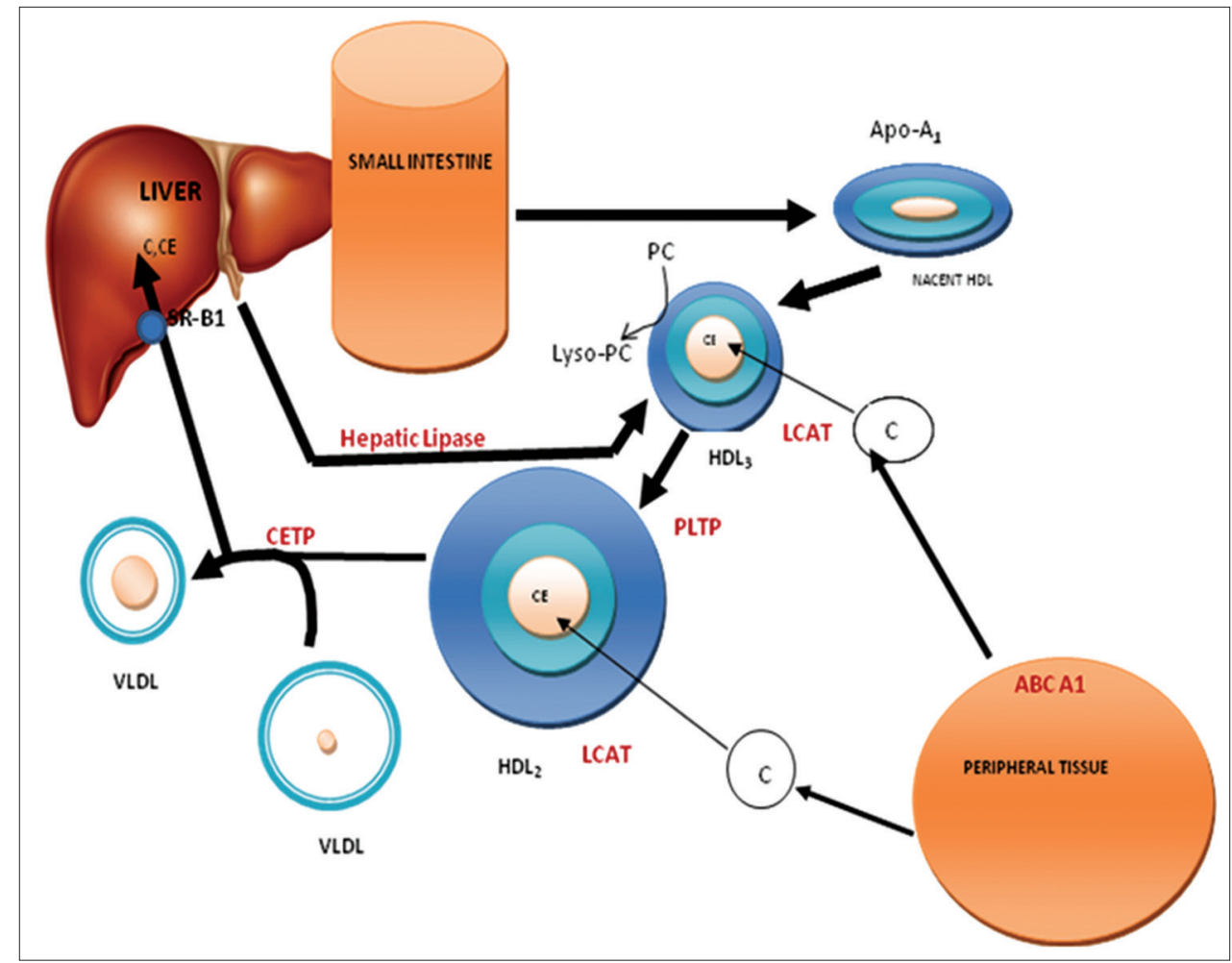

Fig. 5: Reverse cholesterol transport [17,18]. HDL3: High-density lipoproteins-3, HDL2: High-density lipoproteins-2, VLDL: Very low-density lipoproteins, C: Cholesterol, CE: Cholesterol esters, SR-B1: Scavenger receptor class B Type 1, Apo-A1-Apolipoprotein, ABC-A1 - ATP-binding membrane cassette transporter, PC: Phosphatidylcholine, Lyso PC: Lysophosphatidylcholine, LCAT: Lecithincholesterol acyltransferase, PLTP: Phospholipid transfer protein, CETP: Cholesterol ester transfer protein

SREBP regulates the expression of the LDL receptor and cholesterol synthesis. When stores are abundant SREBPs are bound to the ER by SREBP-cleavage-activating protein (SCAP) and insulin-induced gene 1 $[21,22]$. When sterol levels fall, SCAP allows site-1 cleavage to occur by site- 1 protease, which separates the functional domain of SREBP from the regulatory domain [23]. SREBP moves to the Golgi where site-2 protease cleavage frees the NH2-terminal bHLH-Zip domain and allows for its migration to the nucleus for transcriptional regulation of target genes [24]. There are three main SREBPS encoded by 2 genes: SREBP-1a and SREBP-1c are produced from a single gene and SREBP-2 is produced from a separate gene [24]. SREBP-1 has been shown to regulate genes involved in fatty acid metabolism, while SREBP-2 is known to regulate genes involved in cholesterol metabolism [25]. The SREBP-2 gene is regulated by TH. In a study on rats, it was 


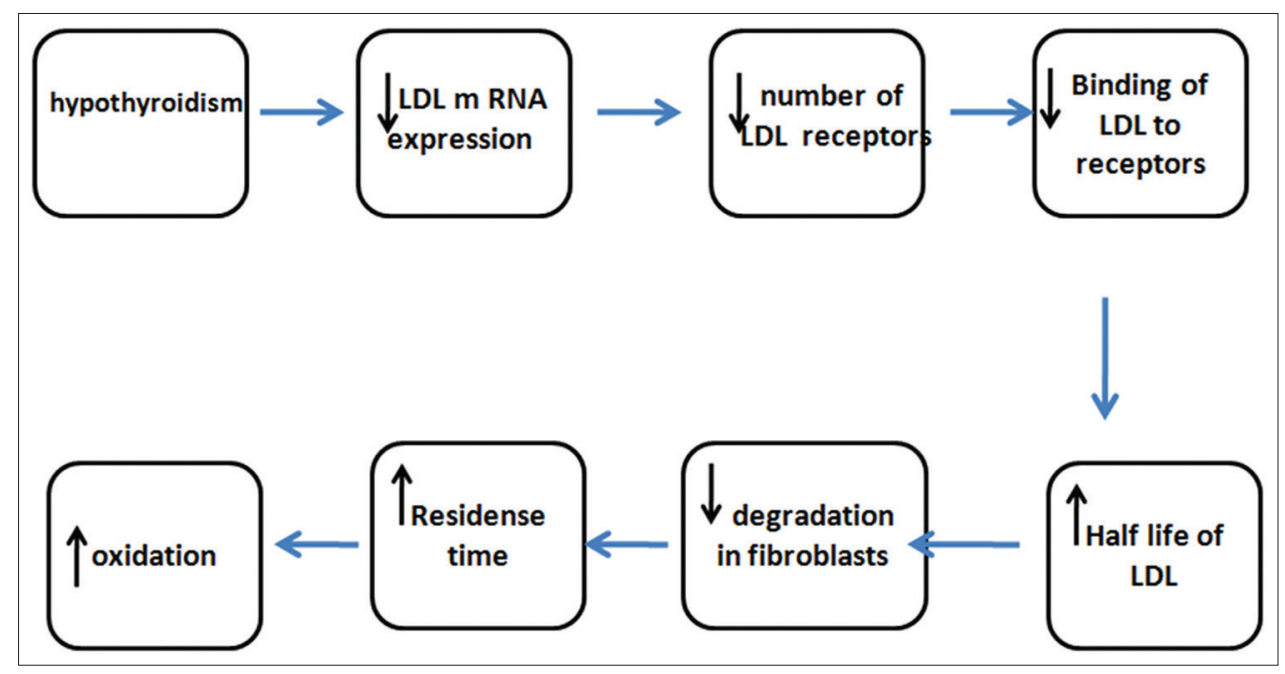

Fig. 6: Pathophysiology of hypothyroidism

demonstrated that the decreased LDL receptor and increased serum cholesterol levels associated with hypothyroidism were secondary to the TH effects on SREBP-2 [26].

\section{Pathophysiology of hypothyroidism}

In hypothyroidism, the expression of LDL mRNA is reduced, leading to decrease in the number of LDL receptors. This in turn leads to decreased binding of LDL to LDL receptors, which results in an increase in the half-life of LDL levels, reduced degradation of LDL in the fibroblasts, enhanced residence time in serum, and susceptibility to oxidation [14] (Fig. 6).

\section{Hypothyroidism and lipid metabolism}

THs influence all major metabolic pathways. The well-known action of THs is an increase in basal energy expenditure obtained by acting on carbohydrate, protein, and lipid metabolism [26]. In addition, thyroid disorders, including overt and SCH, significantly alters the lipid profile and promote CVD [11]. TH is known to play a role in regulating the synthesis, metabolism, and mobilization of lipids [4]. Alterations in thyroid functions also result in a change in the composition and transport of LPs $[2,9,10]$. Studies consistently demonstrate elevated levels of serum total cholesterol (TC), LDL-C, apolipoprotein B, lipoprotein (a), and possibly TGs in individuals with subclinical and overt hypothyroidism, all of which are reversible with levothyroxine therapy $[4,27,28]$. Increased serum TG levels are associated with proatherogenic changes in LPs, for instance, reduction of cardioprotective HDL and generation of small dense LDL (sdLDL) [14].

THs induce the enzyme HMG-CoA reductase, which regulates the first step in cholesterol biosynthesis [19]. In hypothyroid patients, despite the reduced activity of HMG-CoA reductase, there is often an increase in the serum TC concentration, mainly due to raised levels of serum LDL-C and IDL cholesterol $[4,14,16,27-30]$. This is caused by a reduction in LDL receptors [16] and the diminishing control by T3 over SREBP-2, crucial for the expression of LDL receptor [14]. THs increase the synthesis and mobilization of TGs stored in the adipose tissue, which is reflected as an increase in the concentration of non-esterified fatty acids [31]. THs stimulate the lipoprotein lipase (LPL), which catabolizes the TG-rich LPs, and the HL, which hydrolyzes $\mathrm{HDL}_{2}$ to $\mathrm{HDL}_{3}$ and contributes to the conversion of IDL to LDL and in turn LDL to sdLDL [4,29,32]. HL also serves as a ligand that facilitates the uptake of LP by cell-surface receptors and proteoglycans, thus directly affecting cellular lipid delivery [32]. Furthermore, $\mathrm{T}_{3}$ upregulates LDL receptors by controlling the LDL receptor gene activation. The $\mathrm{T}_{3}$-mediated gene activation is done by the direct binding of $\mathrm{T}_{3}$ to specific TH response elements [29]. In hypothyroidism, decreased LPL activity leads to decreased clearance of TG. Therefore, hypothyroid patients may also present with elevated TG levels associated with increased levels of VLDL and occasionally fasting chylomicronemia [31,33-35].

The main role of CETP is exchange of cholesterol esters from $\mathrm{HDL}_{2}$ to the VLDL and TGs in the opposite direction [9]. The activity of CETP is increased by THs ultimately affecting the HDL metabolism. Hypothyroidism patients usually exhibit elevated levels of HDLCmainly due to increased concentration of HDL2 particles resulting from decreased HL activity [36] and also shows decreased activity of CETP which results in the reduced transfer of cholesterol esters from HDL to VLDL [30]. In addition, hypothyroid patients also show increased lipoprotein (a) (LP [a]) levels, which acts as an additional contributing factor for CVD risk [36-38]. However, thyroxine therapy, in a thyrotropin (TSH)-suppressive dose, usually leads to a considerable improvement of the lipid profile [9].

\section{THs and cardiovascular hemodynamic}

THs are known to play a crucial role in cardiac hemodynamics and both hypo- and hyperthyroidism may develop cardiac abnormalities [39]. In addition, hypothyroidism is also associated with antioxidant imbalance. Increased production of free radicals and decreased antioxidant defense mechanism leads to oxidative stress [40]. Hypothyroidism increases the oxidation of plasma cholesterol mainly because of an altered pattern of binding, which presents a substrate for the oxidative stress [9]. THs cause vascular smooth muscle relaxation resulting in decreased arterial resistance and diastolic blood pressure. Overt hypothyroidism is associated with increased systemic vascular resistance, diastolic hypertension, decreased cardiac contractility, decreased cardiac output, and accelerated atherosclerosis and CAD [41]. In addition to this, another biochemical parameter, the plasma concentration of homocysteine, which acts as an independent risk factor for CVD, was also found to be high in hypothyroid patients $[42,43]$. In addition to all these risk factors, the abnormalities of lipid metabolism associated with overt hypothyroidism contribute to the development of atherosclerotic CAD.

\section{CONCLUSION}

THs play an important role in the regulation of lipid metabolism. Hypothyroidism is linked with an increase in cholesterol, TGs, and LDL-C levels. THs also associated with endothelial dysfunction, normal/ depressed systolic function, left ventricular diastolic dysfunction at rest, and systolic and diastolic dysfunction on effort. The atherogenic lipid profile along with other predisposing factors may contribute to the CAD.

\section{CONFLICTS OF INTEREST}

There are no conflicts of interest. 


\section{REFFERENCES}

1. Roberts CG, Ladenson PW. Hypothyroidism. Lancet 2004;363:793-803.

2. Canaris GJ, Manowitz NR, Mayor G, Ridgway EC. The colorado thyroid disease prevalence study. Arch Intern Med 2000;160:526-34.

3. Almandoz JP, Gharib H. Hypothyroidism: Etiology, diagnosis, and management. Med Clin North Am 2012;96:203-21.

4. Pearce EN. Hypothyroidism and dyslipidemia: Modern concepts and approaches. Curr Cardiol Rep 2004;6:451-6.

5. Salvatore D, Davies TF, Schlumberger M, Hay ID, Larsen PR. In: Melmed S, Polonsky KS, Larsen PR, Henry MK, editors. Thyroid Physiology and Diagnostic Evaluation of Patients with Thyroid Disorders. Philadelphia, PA: Williams Text Book Of Endocrinology; 2011. p. 327-361.

6. Inada M, Kasagi K, Kurata S, Kazama Y, Takayama H, Torizuka K, et al. Estimation of thyroxine and triiodothyronine distribution and of the conversion rate of thyroxine to triiodothyronine in man. J Clin Invest 1975;55:1337-48.

7. Salvatore D, Davies TF, Schlumberger MJ, Hay ID, Larsen PR. In: Melmed S, Polonsky KS, Larsen PR, Henry MK, editors. Thyroid Physiology and Diagnostic Evaluation of Patient with Thyroid Disorders. William's Text Book Of Endocrinology. $12^{\text {th }}$ ed. Philadelphia, PA: Saunders; 2011. p. 334-415.

8. Mebis L, van den Berghe G. The hypothalamus-pituitary-thyroid axis in critical illness. Neth J Med 2009;67:332-40.

9. Duntas LH. Thyroid disease and lipids. Thyroid 2002;12:287-93.

10. Friis T, Pederson LR. Serum lipids in hyper and hypothyroidism before and after treatment. Clin Chim Acta 1987;162:155-63.

11. Tsimihodimos V, Bairaktari E, Tzallas C, Miltiadus G, Liberopoulos E, Elisaf M. The incidence of thyroid function abnormalities in patients attending an outpatient lipid clinic. Thyroid 1999;9:365-8.

12. Chait A, Brunzel JD. Acquired hyperlipidemia (Secondary dyslipoproteinemias). Endocrinol Metab Clin North Am 1990;19:259-78.

13. Stone NJ. Secondary causes of hyperlipidemia. Med Clin North Am 1994;78:117-41.

14. Duntas LH, Brenta G. The effect of thyroid disorders on lipid levels and metabolism. Med Clin North Am 2012;96:269-81.

15. Gotto AM Jr. Interrelationship of triglycerides with lipoproteins and high-density lipoproteins. Am J Cardiol 1990;66:20A-3.

16. Moon JH, Kim HJ, Kim HM, Choi SH, Lim S, Park YJ, et al. Decreased expression of hepatic low-density lipoprotein receptor-related protein 1 in hypothyroidism: A novel mechanism of atherogenic dyslipidemia in hypothyroidism. Thyroid 2013;23:1057-65.

17. Harvey RA, Ferrier DR. Cholesterol and steroid metabolism. Unit 3-lipid metabolism. In: Lippincott's Illustrated Reviews. Biochemistry. $5^{\text {th }}$ ed. Philadelphia, PA: JB. Lippincott Company; 2011. p. 219-44.

18. Ohashi R, Mu H, Wang X, Yao Q, Chen C. Reverse cholesterol transport and cholesterol efflux in atherosclerosis. QJM 2005;98:845-56.

19. Espenshade PJ, Hughes AL. Regulation of sterol synthesis in eukaryotes. Annu Rev Genet 2007;41:401-27.

20. Párraga A, Bellsolell L, Ferré-D’Amaré AR, Burley SK. Co-crystal structure of sterol regulatory element binding protein 1a at $2.3 \mathrm{~A}$ resolution. Structure 1998;6:661-72.

21. Adams CM, Goldstein JL, Brown MS. Cholesterol-induced conformational change in SCAP enhanced by insig proteins and mimicked by cationic amphiphiles. Proc Natl Acad Sci U S A 2003;100:10647-52.

22. Sun LP, Li L, Goldstein JL, Brown MS. Insig required for sterolmediated inhibition of scap/SREBP binding to COPII proteins in vitro. J Biol Chem 2005;280:26483-90.
23. Brown MS, Goldstein JL. The SREBP pathway: Regulation of cholesterol metabolism by proteolysis of a membrane-bound transcription factor. Cell 1997;89:331-40.

24. Brown MS, Goldstein JL. A proteolytic pathway that controls the cholesterol content of membranes, cells, and blood. Proc Natl Acad Sci U S A 1999;96:11041-8.

25. Eberlé D, Hegarty B, Bossard P, Ferré P, Foufelle F. SREBP transcription factors: Master regulators of lipid homeostasis. Biochimie 2004:86:839-48.

26. Shin DJ, Osborne TF. Thyroid hormone regulation and cholesterol metabolism are connected through sterol regulatory element-binding protein-2 (SREBP-2). J Biol Chem 2003;278:34114-8.

27. Pucci E, Chiovato L, Pinchera A. Thyroid and lipid metabolism. J Clin Endocrinol Metab 2012;97:326-33.

28. Duntas LH, Wartofsky L. Cardiovascular risk and subclinical hypothyroidism: Focus on lipids and new emerging risk factors. What is the evidence? Thyroid 2007;17:1075-84

29. Liberopoulos EN, Elisaf MS. Dyslipidemia in patients with thyroid disorders. Hormones (Athens) 2002;1:218-23.

30. Rizos CV, Elisaf MS, Liberopoulos EN. Effects of thyroid dysfunction on lipid profile. Open Cardiovasc Med J 2011;5:76-84.

31. Pucci E, Chiovato L, Pinchera A. Thyroid and lipid metabolism. Int J Obes Relat Metab Disord 2000;24 Suppl 2:S109-12.

32. Jha PK, Mandal GK. Study of thyroid profile in geriatric Type-2 diabetics in Jharkhand. Asian J Pharm Clin Res 2017;10:422-4.

33. Santamarina-Fojo S, González-Navarro H, Freeman L, Wagner E, Nong Z. Hepatic lipase, lipoprotein metabolism, and atherogenesis. Arterioscler Thromb Vasc Biol 2004;24:1750-4

34. Al-Tonsi AA, Abdel-Gayoum AA, Saad M. The secondary dyslipidemia and deranged serum phosphate concentration in thyroid disorders. Exp Mol Pathol 2004;76:182-7.

35. Teixeira Pde F, Reuters VS, Ferreira MM, Almeida CP, Reis FA, Buescu A, et al. Lipid profile in different degrees of hypothyroidism and effects of levothyroxine replacement in mild thyroid failure. Transl Res 2008;151:224-31.

36. Pearce EN, Wilson PW, Yang Q, Vasan RS, Braverman LE. Thyroid function and lipid subparticle sizes in patients with short-term hypothyroidism and a population-based cohort. J Clin Endocrinol Metab 2008;93:888-94.

37. de Bruin TW, van Barlingen H, van Linde-Sibenius Trip M, van Vuurst de Vries AR, Akveld MJ, Erkelens DW, et al. Lipoprotein(a) and apolipoprotein B plasma concentrations in hypothyroid, euthyroid, and hyperthyroid subjects. J Clin Endocrinol Metab 1993;76:121-6.

38. Tzotzas T, Krassas GE, Konstantinidis T, Bougoulia M. Changes in lipoprotein(a) levels in overt and subclinical hypothyroidism before and during treatment. Thyroid 2000;10:803-8

39. Gupta G, Sharma P, Kumar P, Sharma R. Cardiovascular risk in patients with mild to severe subclinical hypothyroidism. Asian J Pharm Clin Res 2016;9:183-5.

40. Bhimte B, Agrawal BK, Sharma VK, Chau SS. Oxidative stress status in hypothyroid patients. Biomed Res 2012;23:286-8.

41. Klein I, Danzi S. Thyroid disease and the heart. Circulation 2007; 116:1725-35

42. Morris MS, Bostom AG, Jacques PF, Selhub J, Rosenberg IH. Hyperhomocysteinemia and hypercholesterolemia associated with hypothyroidism in the third US national health and nutrition examination survey. Atherosclerosis 2001;155:195-200.

43. Souad L, Cherfa A, Dalila N. The effects of homocysteine on plasma biochemical parameters and aortic matrix metalloproteinases activities. Int J Pharm Pharm Sci 2015;7:459-62. 